\title{
Data Dissemination Protocols for Reprogramming Wireless Sensor Networks: A Survey
}

\author{
G. V. Keswani \\ Department of Computer Application. Shri Ramdeobaba College of Engineering and Management, Nagpur, India \\ Email: keswanigv@rknec.edu
}

Received: 20 ${ }^{\text {th }}$ September 2018, Accepted: $11^{\text {th }}$ October 2018, Published: $31^{\text {st }}$ October 2018

\begin{abstract}
The wireless sensor network (WSN) is made up of a huge number of programmed sensor nodes spatially scattered to monitor and control environmental conditions. Reprogramming is crucial to upgrade the changes needed and to repair the bugs found in nodes once it is deployed in a hostile situation. Once sensor nodes are deployed then it is not possible to reprogram such nodes manually. Therefore, the sensor nodes are reprogrammed automatically and remotely lacking manual interference. At present several data dissemination protocols have been designed and play important role in dissemination of configuration parameters, program code, queries, commands, bulk data or code packets etc. This paper presents a survey of existing data dissemination protocols employed for reprogramming WSNs and discusses research related issues which provides a broad vision for future research in WSNs domain.
\end{abstract}

\section{Keywords \\ Data Dissemination, Nodes, Protocols, Reprogramming, Wireless Sensor Networks}

\section{Introduction}

The applications of Wireless Sensor Networks (WSNs) day by day increases, there is also rise in population of nodes and the requirement to be able to succeed them remotely becomes more efficient [1]. Such systems must regularly function unattended for longer periods of time which leads necessity for reprogramming the sensor nodes, whereas maintenance and updating of code are required for improving security along with adjustments to the situation once deployed. In evolving WSNs which contain hundreds or even thousands of nodes, one-by-one reprogramming them necessitates both physical admittance to every node and contains an enormously time-taking practice, hampering any real-time reprogramming lacking human attention. The facility to enhance latest functionality or make software maintenance deprived to physically arrive at every node is already a vital service.

Transmitting the code image that the sensor node will perform over the air signifies an actual task due to the improved trustworthiness required (since here the whole image must be received by each node) though the wireless medium is intrinsically untrustworthy. Additionally, owing to inadequate memory, the involving nodes should not preserve big programming code files and because of restricted resource processing the complexity of the implemented code distribution approaches need to be cautiously evaluated to confirm feasibility. If the code image is not able to be adjusted into one packet, it needs to be in steady storage till the transfer is over, at this instant the node can be reprogrammed safely. Since the transmission attribute of the wireless medium should be utilized to pay for the low communication throughput, proper steps required to be followed in case of missing segments (e.g. owing to errors or collisions) either when all or just one of the nodes which receives data has not received a packet successfully. Henceforth, a real challenge has to face in the design of a trustworthy data dissemination protocol for broadcasting big data objects from one or many source nodes to different nodes through a multi-hop, wireless sensor network. The security, scalability and reliability issues in a wireless sensor networks become a major issue as the reprogramming process is potentially vulnerable to serious attacks. The performance requirements of a sensor node with respect to reprogramming protocols consist of resource efficiency, overhead of computation, communication energy and memory. On the parallel grounds the time needed in propagation and implementation of reprogramming protocol should also be considerably less.

The rest of this paper is organized as follows. Section 2 comprises of related work in the domain of wireless sensor network reprogramming and data dissemination protocols. Section 3 consists of a tabular representation of the existing data dissemination protocols for reprogramming with their traits. Section 4 concludes this paper. Section 5 shows references included in this paper.

\section{Brief Review of Data Dissemination Protocols}

This section presents a survey on data dissemination protocols exists for reprogramming wireless sensor networks. 
A reliable bulk data dissemination protocol called CORD [2] has been proposed for disseminating a large data object to the nodes present in wireless sensor network. In contrast to Deluge [3] that pursues a centralized reprogramming approach within which solely the base station will include in data dissemination, CORD pursues a distributed reprogramming wherein several nodes in the sensor network directly include in data dissemination. Like Deluge CORD conjointly pursues the concept of pipelining. The prime criterion of Deluge is to minimize the latency of object dissemination, whereas the main target of CORD is to consume less energy. CORD uses a two-phase approach to acquire its goals. In the first phase, the sensor nodes within their network that grasp a reliable link to the close by nodes are called core nodes. After that the data would be propagated to the same core nodes. In the second phase, the data is propagated from core nodes to non-core nodes within the sensor network. This scheme diminishes the count of messages transmit across the network that successively minimizes the consumption of energy, collision, and contention within the network. Additionally, CORD employs a coordinated sleep schedule method wherein nodes that are not included in transmission of data shifts to the sleep mode to further diminish the consumption of energy. CORD's second phase becomes lengthy for disseminating additional count of pages because there is a significant rise in the count of packets to be transferred from core nodes to non-core nodes. The performance of CORD has been evaluated experimentally on each an indoor and outdoor wireless sensor network testbed and through essential simulations.

A basic service in sensor networks is Over-the-air programming (OAP) [4] that depends upon trustworthy broadcast for efficient dissemination. Mainly, current OAP protocols rapidly degrade in their performance with high node density and even more with high loss of packet. OAP protocols rely on the utilization of rateless coding to remove the necessity to spread the control information regarding which packets necessitate retransmission. Due to this approach, a node must solely receive an adequate count of different, encoded packets to recover a program which has been transmitted. Rateless codes should be implemented in the resource limited surroundings of a wireless sensor network which necessitates the design of proficient methods to diminish computational complexity, memory overhead, and latency. To demonstrate the effectiveness of using rateless codes for OAP, we propose and implement two new protocols. Two OAP protocols has been proposed and implemented to show the effectiveness of applying rateless analogs for replacing the data transfer techniques of existing OAP Deluge. The first OAP protocol titled rateless Deluge remarkably varies the transmit system of the Over-the-air programming Deluge protocol to permit the rateless transmission of program images. The second OAP protocol titled ACKless Deluge supplements the rateless Deluge protocol by employing a packet level FEC technique which targets at suspending the necessity for additional control packets. ACKless Deluge spread other encoded packets to prevent the necessity of retransmissions of packets.

In this paper [5], a lightweight and density aware reprogramming protocol called ReXOR has been anticipated for WSNs engaging XOR. It supports XOR encoding in the retransmission phase to diminish the expenses occurred in communication. XOR gives improved performance than Deluge in lossy and sparse networks thus substantially lead to a standard reprogramming protocol for WSNs. However, prior reprogramming protocols based on coding aren't lightweight with respect to computation overhead. Precisely, Rateless Deluge [4], and SYNAPSE [6] need Gaussian elimination for decoding but Gaussian elimination is costly with respect to computation time. As related to previous reprogramming protocols depending on coding, it provides two relevant features. Firstly, it's computationally lightweight as compared to existing reprogramming protocols depending on coding engaging Fountain Codes or Random Linear Codes. Secondly, it uses the adaptive performance in a density-aware way by assessing the interpage wait time. Subsequently, it achieves smart performance in every sparse and dense network. Execution of ReXOR is principally trusted on TinyOS wherein its performance is assessed. Experimental outcomes specify that ReXOR is undoubtedly lightweight compared to earlier reprogramming protocols depending on coding related to overhead of computation. Additionally, the outcomes indicate that compared with Deluge and a standard coding-based reprogramming protocol, Rateless Deluge, ReXOR accomplishes smart network-level performance in each dense and sparse network.

Some reprogramming protocols [7] depend on the centralized approach wherein merely the base station has the control to initialise reprogramming for the nodes present in WSNs. Distributed reprogramming provides better approach than centralized approach. Distributed reprogramming permits numerous authorized network users to directly and at the same instant initialise reprogramming for sensor nodes in the absence of base station. Here, network owner should be able to allocate different privilege required for reprogramming nodes to different network users. Thus, the author develops Secure and Distributed Reprogramming Protocol called SDRP which is the foremost distributed reprogramming protocol for WSNs. SDRP expands Deluge to be a safe protocol. SDRP utilizes identity-based cryptography approach to protect the reprogramming and to diminish communication and storage needs of each sensor node. The security properties of SDRP protocol has been demonstrated with the help of theoretical analysis.

A trustworthy bulk data dissemination protocol titled SURF [8] which selectively adopts flooding and negotiation approaches correctly. The Negotiation process approves NACK messages to provide assurance for maintaining the consistency of data transmission but result in the overhead disturbance happens throughout dissemination. On the other hand, every node is accountable to transmit the similar packets $\mathrm{N}$ times in the flooding approach. There is no assurance for reliable data dissemination in flooding. When flooding has been employed to dense networks it begins contention related 
problems and redundancy collision which takes large time to get over. SURF assimilate a reliable structure to choose dynamically the best transit point to move over amid two approaches which require less completion time in one hop and to choose the fittest approach amid the two (Negotiation \& flooding), SURF predicts the time efficiency of every strategy to smoothen the technique of data dissemination. SURF concludes the development of data dissemination in less amount of time irrespective of the circumstance that extra packets transferred result in savings of energy. To further reduce the intake of energy, the sleep scheduling techniques can also be trusted on.

In [9], the author proposed Correlated Dissemination protocol named CD, a bulk data dissemination protocol which relies on link correlation and link qualities. Firstly, it employs link correlation to improve the dissemination performance and secondly it dynamically and selectively utilizes rateless codes once the link correlation is essentially weak. In general, CD is made up of four phases. In Initialization phase, all the participating sensor nodes acquire hop counts and then transmit and exchange essential link information to enhance the routing capabilities of the nodes. In Correlated tree construction phase, consider link information which get collected in initialization phase to improve performance of dissemination, each participating node built a correlated tree in a distributed way. In Coding decision phase, every node constructs a model to evaluate the coding and non-coding techniques performance to evaluate the transmission delay within the group. This helps to select or reject rateless codes for coding. In Data Dissemination phase, prioritized REQ mechanism along with incorporates the coordinated schedules is used so that node request initially with the poorest link quality. This ensures reliability and efficiency of dissemination in the group. Every node initiate dissemination as per the coding or non-coding that's predefined within the coding decision phase. The Correlation Dissemination greatly enhances the performance of dissemination with respect to transmission cost, energy consumption, latency, and completion time.

\section{Discussion}

Table 1 summarizes the traits of the data dissemination protocols described in preceding section. Some research issues can be found as per the previous survey [10].

Whole to pipeline: If new program is bigger in size then attempting for sending the complete program at once becomes slower and error can be introduced in this process. Reprogramming based on pipeline involves division of a huge program into many smaller segments, and the segments are transferred one by one thus leading to a large throughput. Even if there is a fault it can be recovered easily when retransmission is involved, and this process is also cheaper as compared to the huge program when forwards as whole.

All to selective: Previously most solutions do not permit control over whenever a new code or change is required to be distributed, and hence installed to every node present in a wireless sensor network. Two reasons are enough to make it less flexible. Firstly, new programs which aren't included in present tasks will be installed to some other nodes that leads to resource wastage and finally create inefficiency. There may be possibility for several applications to be able to executable at same time in a WSN. The concept of selective reprogramming permits to select merely part of the node set which needs to be reprogrammed for making it efficient obviously and more flexible. By explicitly assigning node IDs, selection becomes static or dynamic by evaluating some criteria at runtime on every single node.

Delivery Scheme: An additional change in reprogramming schemes is delivery of entire code image to difference-based updating. The difference-based updating technique must send only binary differences to nodes, hence is more energy efficient and faster. Entire code-based delivery involves the general reprogramming facility and difference-based scheme should be employed to send differences amid versions.

Idle listening to adaptive sleeping: In Idle listening every node waits for incoming packets even if at an instant a small portion of the complete Wireless Sensor Network is involved in transmission. Idle listening is observed in dissemination which relied on pipeline concept. In reprogramming, idle listening is a dominant means for wastage of energy, and that needs to be eliminated. Adaptive sleeping involves a scheme in which a node goes into sleep state when its nearby nodes are transmitting data which is not required and hence becomes energy efficient.

Plain to hierarchical: In a hierarchical reprogramming scheme, software updates are initially transferred to nodes present in the upper layer of the node hierarchy, and then they proliferate to nodes present in the lower layers up to end leaves. Some super nodes of stronger platforms or normal nodes in same way cover local areas are the middle layer nodes. Nodes present in the middle layers are considered cluster heads in wireless sensor network structure based on cluster. As far as scalability is concerned hierarchical reprogramming is superior as compared to plain based scheme, when the extent of wireless sensor network becomes larger, hierarchical reprogramming is comparatively more effective.

Redundant to request: Several dissemination protocols utilize broadcast to allocate program codes to a wireless sensor network. When a node may collect numerous unwanted reprogramming data from his nearby nodes then it leads to data redundancy. If a sender is aware of the data which is acknowledged by its receivers, then the data redundancy can be minimized. One of the solutions for data redundancy is a technique based on three-way-handshake, through advertiserequest-data transferring message amongst neighbors. 


\begin{tabular}{|l|l|l|l|l|l|l|}
\hline $\begin{array}{l}\text { Data Dissemination } \\
\text { Protocol }\end{array}$ & $\begin{array}{l}\text { Whole } \\
\text { /Pipeline }\end{array}$ & $\begin{array}{l}\text { All } \\
\text { /Selective }\end{array}$ & $\begin{array}{l}\text { Delivery } \\
\text { Scheme }\end{array}$ & $\begin{array}{l}\text { Idle Listening } \\
\text { /Adaptive Sleeping }\end{array}$ & $\begin{array}{l}\text { Plain } \\
\text { /Hierarchical }\end{array}$ & $\begin{array}{l}\text { Redundant } \\
\text { / Request }\end{array}$ \\
\hline CORD [2] & pipeline & all & entire code & adaptive sleeping & hierarchical & request \\
\hline $\begin{array}{l}\text { RATELESS } \\
\text { DELUGE [4] }\end{array}$ & whole & all & difference-based & idle listening & hierarchical & request \\
\hline RE-XOR [5] & pipeline & all & difference-based & idle listening & plain & request \\
\hline SDRP [7] & whole & selective & difference-based & idle listening & hierarchical & redundant \\
\hline SURF [8] & pipeline & selective & difference-based & adaptive sleeping & plain & request \\
\hline CD [9] & pipeline & selective & difference-based & idle listening & hierarchical & redundant \\
\hline
\end{tabular}

Table1. Traits of Data Dissemination Protocols Analyzed for Reprogramming WSNs.

\section{Conclusion}

Wireless Sensor Networks is an open and wide domain for doing research in networking, which is gradually being installed for monitoring and controlling applications. This demands the requirement for spreading data and code efficiently via wireless links to sensor nodes to reprogram them to fulfil the present desires of the application. This is accomplished by proper employing data dissemination protocols. In this paper, a brief survey work is done on the existing data dissemination protocols for reprogramming WSNs and their research related issues were discussed. These protocols have a common goal of trustworthy data dissemination for reprogramming WSNs. This gives a broad vision for doing future research in WSNs domain.

\section{References}

[1] Helen C. Leligou, Christos Massouros, Eleftherios Tsampasis, Theodore Zahariadis, Dimitrios Bargiotas, Konstantinos Papadopoulos, Stamatis Voliotis "Reprogramming wireless sensor networks", in International Journal of Computer Trends and Technology, Vol.1, 2011.

[2] Leijun Huang; Setia, S., "CORD: Energy-Efficient Reliable Bulk Data Dissemination in Sensor Networks", in IEEE INFOCOM'08, 2008.

[3] J. W. Hui and D. Culler, "The dynamic behavior of a data dissemination protocol for network programming at scale", in ACM SenSys, 2004.

[4] A. Hagedorn, D. Starobinski, and A. Trachtenberg, "Rateless Deluge: Over-the-air programming of wireless sensor networks using random linear codes," in IEEE IPSN'08, 2008.

[5] Wei Dong, Chun Chen, Xue Liu, Jiajun Bu, Yi Gao, "A Lightweight and Density-Aware Reprogramming Protocol for Wireless Sensor Networks,” IEEE Transactions on Mobile Computing, Vol.10, 2011.

[6] Michele Rossi, Giovanni Zanca, Luca Stabellini, Riccardo Crepaldi, Albert F. Harris III and Michele Zorzi, "SYNAPSE: A Network Reprogramming Protocol for Wireless Sensor Networks using Fountain Codes", in IEEE SECON '08, 2008.

[7] D. He, C. Chen, S. Chan, and J. Bu, "SDRP: A secure and distributed reprogramming protocol for wireless sensor networks," IEEE Transactions on Industrial Electronics, Vol. 59, 2012.

[8] Xiaol ong Zheng Jiliang Whe, Wei Dong; Yuan He, Yunhao Liu, "Survival of the Fittest: Data Dissemi nat i on with Selective Negot iati on in Wreless Sensor Net works", in MSS' 13, 2013.

[9] Zhi wei Zhao Wei Dong, Ji aj un Bu, Yu Gu, Chun Chen, "Li nk Correlati on Avare Dat a Di ssemi nat i on in Wrel ess Sens or Net works”, I EEE Transact i ons on Industrial El ect roni cs, Vol. 62, 2015.

[10] Jun-Zhao Sun, "Dissemination Protocols for Reprogramming Wireless Sensor Networks: A Literature Survey", in IEEE Fourth International Conference on Sensor Technologies and Applications, 2010. 